\title{
A Rare Branching Pattern of a Middle Mesenteric Artery Supplying the Head of the Pancreas and the Transverse Colon
}

\author{
Dionysios Venieratos $^{1}$, Gregory Tsoucalas ${ }^{2}$, Eleni Panagouli ${ }^{1}$
}

${ }^{1}$ Department of Anatomy, Medical School, National and Kapodistrian University of Athens, Greece, ${ }^{2}$ History of Medicine, Department of Anatomy, Democritus University of Thrace, Alexandroupolis, Greece.

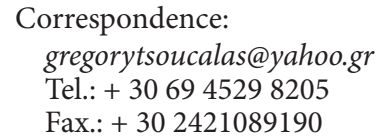

Received: 1 June 2018

Accepted: 24 October 2018

Key words: Inferior Pancreaticoduodenal Artery - Middle Colic Artery - Abdominal Aorta - Superior Mesenteric Artery.
Objective. The appearance of a middle mesenteric artery is a rare branching variation, with an incidence lower than $0.1 \%$. Our case reports such an anatomical artery pattern which was discovered in a male Caucasian cadaver during routine educational dissection. This alternation is seldom encountered and may trouble diagnostic and surgical interventions. Case Report. The case of a Middle Mesenteric Artery is described, as a vessel originating from the Abdominal Aorta $3.3 \mathrm{~cm}$ below the origin of the Superior Mesenteric and $3.8 \mathrm{~cm}$ above the origin of the Inferior Mesenteric Artery. The middle mesenteric artery, directed upwards, gives two branches for supplying blood to the head of the pancreas. We first mention the thinner branch, corresponding to the posterior one of the inferior pancreaticoduodenal artery, and second the thicker branch corresponding to the anterior branch of the Inferior Pancreaticoduodenal Artery. The main artery continues its course between the two sheets of the mesocolon to supply the transverse colon, thus substituting the normal colic artery. Conclusion. The occurrence of a Middle Mesenteric Artery constitutes a very rare anatomic variation. We present, to our knowledge, the first case described where the Middle Mesenteric Artery provides blood to the pancreas.

\section{Introduction}

The Middle Colic Artery (MCA) normally emerges from the Superior Mesenteric Artery, just inferior to the uncinate process of the pancreas, and anteriorly to the third part of the duodenum. The artery may branch either separately or as a common trunk with the Right Colic Artery (1-2). The Middle Mesenteric Artery (MMA) is defined as a vessel which originates directly from the abdominal aorta. In these cases, the vessel arises between the Superior (SMA) and the Inferior Mesenteric Arteries (IMA) and mainly supplies the transverse, and sometimes the descending or ascending colon (3-6). The MMA is considered to be a very rare variation, with an incidence lower than $0.1 \%$ (5).

The Inferior Pancreaticoduodenal Artery (IPA) arises more commonly from the SMA (posterior, left and right lateral or anterior side) and supplies the pancreatic head $(1,7)$. This artery may present with various anatomic points of emergence. Thus, it might emerge a) from a common trunk with the first jejunal branch (20-64.7\% of cases), b) from a common trunk with the dorsal pancreatic artery (6-8\% of cases), c) from a common trunk with the second jejunal artery ( $2 \%$ of cases), d) from the right acces- 
sory hepatic artery (the incidence has not been estimated), e) more rarely from a common trunk with the first two or three jejunal arteries, f) from the transverse pancreatic artery, g) from the MCA and h) from an accessory $\operatorname{MCA}(7,8)$.

\section{Case Report}

We report an anatomical variation of an MMA which was discovered in a male Caucasian cadaver (deceased at the age of 66), during routine educational dissection at our Anatomy Department. The cadaver derived from a body donation with informed consent, written and signed (with signature authentication) by the donor himself. For our review of the literature, we conducted a thorough search in the PubMed database, using the words "Middle Mesenteric Artery" and "Inferior Pancreaticoduodenal Artery" as key terms. In the present case report, we describe a MMA, which provides two branches to the head of the pancreas before its termination as an MCA.

The branches of the abdominal aorta were carefully dissected. The stomach and the pancreas were also dissected and mobilized to expose the celiac trunk, the SMA and their branches. The Inferior Pancreaticoduodenal Artery and the MCA were not detected as one of the branches of the $\mathrm{Su}$ perior Mesenteric Artery, as expected. Then the transverse colon and mesocolon were retracted in order to reveal the IMA and its branches. An MMA was observed originating from the Abdominal Aorta between the Superior and Inferior Mesenteric Arteries, $3.3 \mathrm{~cm}$ distal to the SMA and $3.8 \mathrm{~cm}$ proximal to the inferior one. This artery supplied

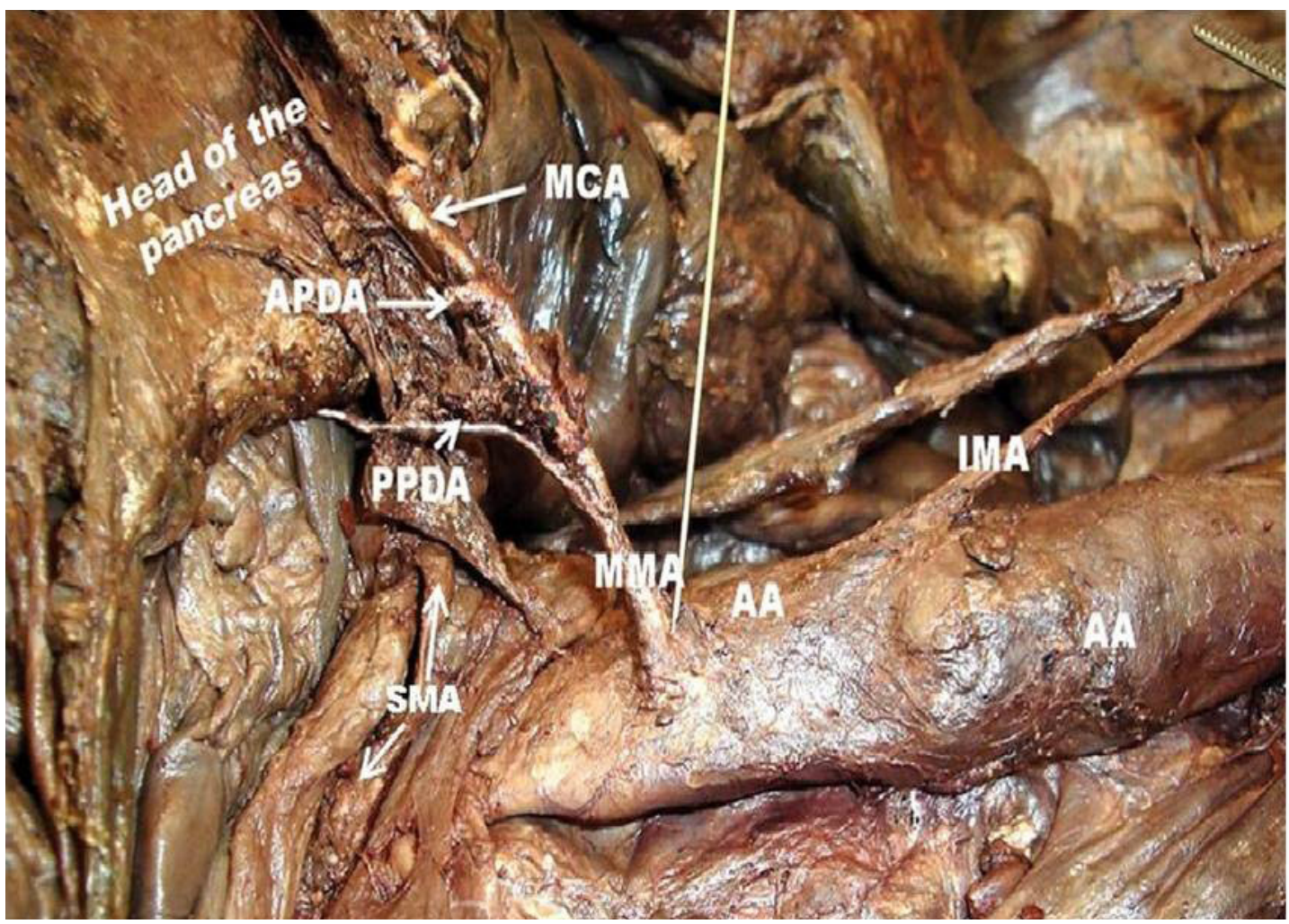

Figure 1. A rarely encountered Middle Mesenteric Artery. MMA=The Middle Mesenteric Artery; $A A=A b d o m i n a l$ Aorta; IMA=Inferior Mesenteric Artery; APDA=Anterior Pancreaticoduodenal Artery; PPDA=Posterior Pancreaticoduodenal Artery; SMA=Superior Mesenteric Artery; MCA=Middle Colic Artery; IVC=Inferior Vena Cava. 
the transverse colon as well as the head of the pancreas, as follows: It produced a rather thin (proximal) branch to the posterior surface of the head of the pancreas at first (after a course of $1.8 \mathrm{~cm}$ ), then it coursed in close contact with the pancreatic head, supplying a second (rather thick) branch (distal), 2.6 $\mathrm{cm}$ after the proximal one (Figure 1). The first branch, after a short course, was anastomosed with branches of the Superior Pancreaticoduodenal Artery, while the second one entered the head of the pancreas from its anterior surface. Thereafter, it continued its route in the transverse mesocolon to reach the transverse colon, as the MCA does (Figure 1). The two branches thus detected were considered to substitute the anterior (the thick one) and posterior (the thin one) branches of the Inferior Pancreaticoduodenal Artery (Figure 1). The usual Colic and Pancreaticoduodenal Branches of the Superior Mesenteric Artery were absent, whereas it gave rise to the usual ileocolic, jejunal and ileal branches. The rest of the arterial pattern of the gut was normal, as anatomically expected.

\section{Discussion}

The mere existence of an unpaired arterial branch between the Superior and Inferior Mesenteric Arteries is occasionally reported as a case report and should therefore be considered as rare. This branch, whenever it exists, may be named either "Middle Mesenteric", from which the MCA emerges in most cases, or "Middle Colic" originating from the aorta (4). We prefer and propose the term "Middle Mesenteric", as more succinct: it indicates both the position of the artery and the rareness of its existence. Moreover, the term is more collective, as it encompasses all possible further variations. For example, this artery does not always provide a branch to the transverse colon, i.e. a Middle Colic Artery.
Nine such cases of an MMA were found in the literature (Table 1). According to the available data, the first report of such a variation was made by Benton and Cotter in 1963. They described an "anomalous mesenteric artery" in a cadaver, which emerged from the aorta between the superior and inferior mesenteric arteries. The artery was named as "a duplication of the Inferior Mesenteric Artery". In this case the middle colic branch of the SMA did not exist (9). In 1987, Lawdahl and Keller reported an angiogram, in which a similar artery was revealed and also had a similar course, supplying the splenic flexure of the colon. This time the middle colic branch of the SMA was present. The authors called it a "Middle Mesenteric Artery" for the first time and may claim its denomination (10). Yoshida et al. in 1993 described another case of an MMA, also revealed during an angiography. This one had two branches, one right branch anastomosed (within the mesentery) with the right colic artery, and one left branch that supplied the left part of the transverse colon, the splenic flexure and the proximal descending colon. Only its final and marginal branch communicated with the corresponding branch which derived from the left colic artery (from the inferior mesenteric) (3). Higashi and Hirai reported a cadaveric finding in 1998, in which "the second Superior Mesenteric Artery" (as they called it, although its origin was only $2.3 \mathrm{~cm}$ above that of the inferior mesenteric artery) had five small intestinal arteries supplying the inferior portion of the small bowel, and five large intestinal arteries supplying the cecum, the ascending colon, the transverse colon and the superior portion of the descending colon. The authors argued that this arterial pattern was the cause of a malformation in the nonrotation of the intestinal tracts, also observed in that cadaver (11). Another case of colon malrotation was presented by Kawai et al. in 2006. They also observed an MMA supplying the ascending and the transverse 
colon, anastomosed with the SMA and IMA respectively, through the marginal arteries (12). Dirrigl et al. operated on a patient in 2009 with an inflammatory infrarenal aortic aneurysm and discovered a MMA replacing to a large extent both the SMA and a hypoplastic IMA, since it supplied the distal jejunum, the ileum and the colon (ascending, transverse and descending) (13). Kachlik et al. in 2009 described a case of an MMA supplying the left colic flexure and continuing as a left colic artery (4). Naito et al. in 2011 reported a very rare case where the MMA gave a left testicular artery and then supplied the transverse colon (5). Finally, Milnerowicz et al. in 2012 described a cadaveric specimen, in which they also performed arteriography with a MMA totally and exclusively supplying the transverse colon, behaving thus as an aberrant middle colic artery (6).

Our case describes a MMA, which gave off two branches to the head of the pancreas (anterior and posterior branches of the Inferior Pancreaticoduodenal Artery) and continues its course towards the transverse colon as a middle colic artery. Having in mind the arteries described above, we note that no such case seems to have been reported in the literature. The total number of MMAs revealed either through anatomical dissection or through arteriograms, or even during operations, is extremely small, and a reliable estimation of its frequency is not feasible. Milnerowicz et al. evaluated it as less than $0.1 \%$. Moreover, no credible association of this rare variation with sex or race may be established (6).

The unpaired visceral branches derive from the ventral splanchnic arteries which develop initially as paired vessels (1). In a later stage of the embryonic development these arteries regress and anastomose usually to three main trunks, the Celiac, Superior Mesenteric and Inferior Mesenteric Arteries $(1,6)$. The branches of these arteries develop afterwards, as the viscera descend into the abdomen. The MMA, according to the literature, seems to be an example of additional unpaired artery supplying the midgut and the hindgut $(4,6)$. In our case, the MMA replaces the MCA and gives also rise to the "absent" Inferior Pancreaticoduodenal Artery, which normally supplies the head of the pancreas and usually emerges from the SMA below the MCA. We assume that during embryonic development this persistent additional branch replaced both the two first branches of SMA, supplying not only the gut but the head of the pancreas also. Milnerowicz et al. as well as Kachlik et al. proceeded to a description of any clinical implications present. It was concluded that routine morphological examination of the arterial pattern of the celiac aorta by either convention-

Table 1. Cases of a Middle Mesenteric Artery

\begin{tabular}{ll}
\hline MMA $^{*}$ reference & MMA Description \\
\hline Benton and Cotter in 1963 & Emerge from the aorta...a duplication of the inferior mesenteric artery \\
Lawdahl and Keller in 1987 & Emerge from the aorta a middle mesenteric artery \\
Yoshida et al. in 1993 & A MMA with two branches \\
Higashi and Hirai reported in 1998 & The second superior mesenteric artery \\
Kawai et al. in 2006 & A MMA anastomosed with the SMA ${ }^{\dagger}$ and IMA \\
Dirrigl et al. in 2009 & A MMA replacing to a large extent both the SMA and a hypoplastic IMA \\
Kachlik et al. in 2009 & A MMA supplying the left colic flexure and continuing as a left colic artery \\
Naito et al. in 2011 & The MMA gives a left testicular artery \\
Milnerowicz et al. in 2012 & A MMA as an aberrant middle colic artery \\
\hline
\end{tabular}

*Middle mesenteric artery; †Superior mesenteric artery; ‡Inferior mesenteric artery. 
al angiography, selective angiography, CT, or MDCT angiography, allows the identification of such a variation before scheduled, or emergency surgery so that any complication is avoided in the operating room, or during any other clinical interventions and examination $(4,6)$. Wadhwa and Barua, 2008, also concluded that a pre-operative selective arteriogram, or other available techniques are important to determine any anomalies in the arterial supply of the pancreas prior to performing selective pancreatic resections (14). Such a procedure would be ultimately useful in the case of a patient like ours with this rare variation.

\section{Conclusions}

In a cadaveric specimen we observed an unusual case of an MMA, originating from the abdominal aorta, with two branches which substituted the posterior and anterior branches of the Inferior Pancreaticoduodenal Artery. Our report is most possibly the first to describe this branching. As this kind of vessel is rarely encountered, our case may have a practical impact on diagnosis, or even upon the method and range of surgical treatment.

\section{What is already known on this topic}

The presence of an MMA is considered rare. However, this vessel, arising directly from the aorta, has a broad spectrum of branching variations.

\section{What this study adds}

Our case reports an MMA which gives two branches to the head of the pancreas before its termination as an MCA. This peculiar branching testifies the variety of anatomic possibilities of an MMA.

Acknowledgments: The authors wish to thank Prof. Dr. Sophia Anagnostopoulou for her cooperation.

Authors' contributions: Conception and design: DV and EP; Acquisition, analysis and interpretation of data: DV and EP; Drafting the article: GT; Revising it critically for important intellectual content: DV; Approved final version of the manuscript: DV.

Conflict of interest: The authors declare that they have no conflict of interest.

\section{References}

1. Standring S, Borley NR, Collins P. Gray's anatomy. The anatomical basis of clinical practice. Edinburgh: Elsevier; 2008.

2. Negoi I, Beuran M, Hostiuc S, Negoi RI, Inoue Y. Surgical Anatomy of the Superior Mesenteric Vessels Related to Colon and Pancreatic Surgery: A Systematic Review and Meta-Analysis. Sci Rep. 2018;8(1):4184.

3. Yoshida T, Suzuki S, Sato T. Middle mesenteric artery: an anomalous origin of a middle colic artery. Surg Radiol Anat. 1993;15(4):361-3.

4. Kachlik D, Laco J, Turyna R, Baca V. A very rare variant in the colon supply-arteria mesenterica media. Biomed Pap Med Fac Univ Palacky Olomouc Czech Repub. 2009;153(1):79-82.

5. Naito M, Yi SQ, Terayama H, Hirai S, Qu N, Itoh M. A left testicular artery arising from a middle mesenteric artery. Clin Anat. 2011;24(1):266-7.

6. Milnerowicz S, Milnerowicz A, Taboła R. A middle mesenteric artery. Surg Radiol Anat. 2012;34(10):973-5.

7. Bertelli E, Di Gregorio F, Bertell L, Civeli L, Mosca S. The arterial blood supply of the pancreas: a review. III. The inferior pancreaticoduodenal artery. An anatomical review and a radiological study. Surg Radiol Anat. 1996;18(2):67-74.

8. Negoi I, Beuran M, Hostiuc S, Negoi RI, Inoue Y. Surgical Anatomy of the Superior Mesenteric Vessels Related to Pancreaticoduodenectomy: a Systematic Review and Meta-Analysis. J Gastrointest Surg. 2018;22(5):802-17.

9. Benton RS, Cotter WB. A hitherto undocumented variation of the inferior mesenteric artery in man. Anat Rec. 1963;145:171-3.

10. Lawdahl RB, Keller FS. The middle mesenteric artery. Radiology. 1987;165:371-2.

11. Higashi N, Hirai K. Nonrotation of the midgut with abnormality of the superior mesenteric artery [in Japanese]. Kaibogaku Zasshi. 1998;73(5):529-32.

12. Kawai K, Koizumi M, Honma S, Tokiyoshi A, Kodama K. A case of nonrotation of the midgut with a middle mesenteric artery. Ann Anat. 2006;188(1):13-7.

13. Dirrigl MA, Zimmermann A, Ockert S, Eckstein $\mathrm{HH}$. Middle mesenteric artery arising from an inflammatory infrarenal aortic aneurysm. J Vasc Surg. 2009;49(2):474-7.

14. Wadhwa S, Barua MP. Anomalous middle colic artery originating from common hepatic artery: a case report. Clin Anat. 2008;21(8):798-9. 